\title{
Anisotropic magnetoresistance of GaAs two-dimensional holes
}

\author{
S. J. Papadakis, E. P. De Poortere, and M. Shayegan \\ Department of Electrical Engineering, Princeton University, Princeton, New Jersey 08544, USA. \\ R. Winkler \\ Institut für Technische Physik III, Universität Erlangen-Nürnberg, Staudtstr. 7, D-91058 Erlangen, Germany.
}

(October 28, 2018)

\begin{abstract}
Experiments on high-quality GaAs (311)A two-dimensional holes at low temperatures reveal a remarkable dependence of the magnetoresistance, measured with an in-plane magnetic field $(B)$, on the direction of $B$ relative to both the crystal axes and the current direction. The magnetoresistance features, and in particular the value of $B$ above which the resistivity exhibits an insulating behavior, depend on the orientation of $B$. To explain the data, the anisotropic band structure of the holes and a re-population of the spin-subbands in the presence of $B$, as well as the coupling of the orbital motion to $B$, need to be taken into account.
\end{abstract}

71.30.+h,71.70.Ej,73.50.-h

There has been interest recently in the ground state of a disordered two-dimensional (2D) carrier system. Twenty years ago, scaling arguments and supporting experimental data indicated that at temperature $T=0$ $\mathrm{K}$ such a system must be insulating [1].2]. However, prompted by new data in Si 2D electrons [3] and subsequently multiple different $2 \mathrm{D}$ carrier systems [4], revealing a metallic-like behavior, this question is being revisited both experimentally and theoretically $[5,8]$.

One specific area of interest has been the spin degree of freedom [6,9-12]. Measurements have shown that increasing the spin-orbit induced zero-magnetic-field spinsplitting leads to a more pronounced metallic behavior in GaAs 2D holes [10,11]. References [9, 12 also report that the metallic behavior in this system is related to transport by two spin-subbands. Experiments with an in-plane magnetic field $(B)$ similarly suggest that the effects of spin are important 13 177. On the other hand, for $2 \mathrm{D}$ systems with finite layer thickness recent calculations predict an anisotropic positive magnetoresistance (MR) caused by the coupling of the orbital motion to $B$ [8,18]. The MR is calculated to be larger for $B \perp I$ than for $B \| I$, where $I$ is the current in the sample. Motivated by this prediction, we measure the MR of a high-mobility 2D hole system (2DHS) in a GaAs (311)A quantum well, in a density range such that the $B=0$ sample resistivity $(\rho)$ shows metallic $T$-dependence. We apply an in-plane $B$ parallel to the $[\overline{2} 33]$ and [011] crystal axes, and measure the MR with $I \| B$ and $I \perp B$ for each case. Some characteristics of our data, such as an overall positive MR, are consistent with the predictions of Ref. [8]. However, we observe a striking dependence of the $\mathrm{MR}$, and in particular of the onset of insulating behavior, on the orientation of $B$ relative to the crystal axes. We show that this dependence is linked to the anisotropy of the 2DHS band structure, and a re-population of the spin-subbands with increasing $B$.

The samples are Si-modulation doped GaAs quantum wells grown on (311)A GaAs substrates. Even at $B=0$, these samples exhibit a mobility anisotropy believed to be due to an anisotropic surface morphology (see [19, 20 and references therein). The interfaces between the GaAs quantum well and the AlGaAs barriers are believed to be corrugated, with ridges along the [233] direction which reduce the mobility for $I \|[01 \overline{1}]$. While the metallic behavior has been studied in this system extensively, little attention has been paid so far to the differences between transport along $[01 \overline{1}]$ and $[\overline{2} 33]$. Our sample is patterned with an L-shaped Hall bar aligned along $[01 \overline{1}]$ and $[\overline{2} 33]$ to allow simultaneous measurement of the resistivities along the two directions. The sample has metal front and back gates to control both the 2DHS density $(p)$ and the perpendicular electric field $\left(E_{\perp}\right)$ applied to the well [10,11]. Measurements are done in dilution and pumped ${ }^{3} \mathrm{He}$ refrigerators with $B$ up to $16 \mathrm{~T}$. In the ${ }^{3} \mathrm{He}$ refrigerator, the sample is mounted on a single-axis tilting stage that can be rotated in-situ to change the plane of the 2DHS from perpendicular to parallel to the applied $B$.

Figure 1 (a) demonstrates the high quality of the 2DHS in our sample. The data of Fig. 11 also reveal the mobility anisotropy observed in this sample: at $30 \mathrm{mK}$ and $p=6.3 \times 10^{10} \mathrm{~cm}^{-2}$, we have $\mu_{[01 \overline{1}]}=425,000 \mathrm{~cm}^{2} / \mathrm{Vs}$ and $\mu_{[\overline{2} 33]}=530,000 \mathrm{~cm}^{2} /$ Vs. As illustrated in Fig. 11(b), the $T$-dependence of $\rho$ is also significantly different along the two directions in the density range where the behavior is metallic. The [011] direction typically shows a larger fractional change in $\rho, \rho(T) / \rho(30 \mathrm{mK})$, than the [233] direction, as $T$ is increased 10,11]. This suggests that the scattering mechanisms associated with the two mobility directions have different $T$ dependencies, and that the orientation of $I$ relative to the crystal axes is an important parameter in understanding the data.

Figure 2 shows $\rho$ at $T=0.3 \mathrm{~K}$ as a function of $B$ applied in the plane of the 2DHS. The left (right) column shows data for $I \|[01 \overline{1}](I \|[\overline{2} 33])$, with the in-plane $B$ both parallel and perpendicular to $I$. To obtain these data, on separate cooldowns the sample was mounted with the $[01 \overline{1}]$ or the $[\overline{2} 33]$ crystal axis parallel to the tilt 

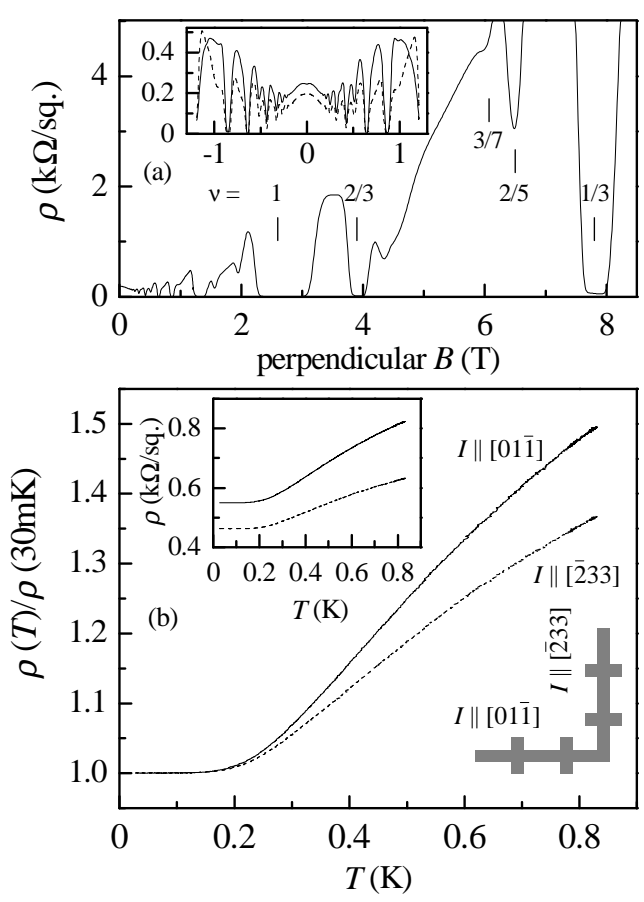

FIG. 1. (a) Resistivity $\rho$ data for magnetic field $B$ perpendicular to the plane of the $2 \mathrm{D}$ hole system with a density $p=6.3 \times 10^{10} \mathrm{~cm}^{-2}$ and current $I \|[\overline{2} 33]$ at $T=30 \mathrm{mK}$. The data exhibits fractional quantum Hall effect at low filling factors $(\nu)$, demonstrating the high quality of the sample. The inset shows low- $B$ data for $I \|[01 \overline{1}]$ (solid trace) and $I \|[\overline{2} 33]$ (dashed trace). (b) $B=0$ temperature-dependence at $p=3.3 \times 10^{10} \mathrm{~cm}^{-2}$, highlighting the difference between [011] (solid) and [233] (dashed) directions. The main figure shows the fractional change in $\rho$ as $T$ is increased, while the inset shows the raw data. The schematic at lower right depicts the Hall bar used for the measurements.

axis. The density $p$ was deduced from the Hall coefficient by measuring the transverse MR in a $B$ perpendicular to the plane of the 2DHS. The stage was then tilted to make the 2DHS plane parallel to the applied $B$, and the MR was measured. The front and back gates were used to change $p$. For $I \|[01 \overline{1}], \rho$ is always larger when $I \perp B$. However, the $I \|[\overline{2} 33]$ data are qualitatively different: at low $B$ and $p$, the $I \perp B$ traces have lower $\rho$, and cross the $I \| B$ traces at higher $B$.

In Fig. 2, there are pronounced qualitative similarities between the dashed traces on the right and the solid traces on the left, and vice versa. To highlight these similarities, in Fig. 3 we show the fractional change in $\rho$, $\rho(B) / \rho(B=0)$, for $B$ along the [2]33] (left column) and [011] (right column) directions. Plotting this way, a striking similarity is evident in the qualitative features of the traces with the same $B$ orientation relative to the crystal axes, even though the $I$ orientations are different. All traces start with a small slope and curve upwards. Then there is an inflection point followed by a reduction in

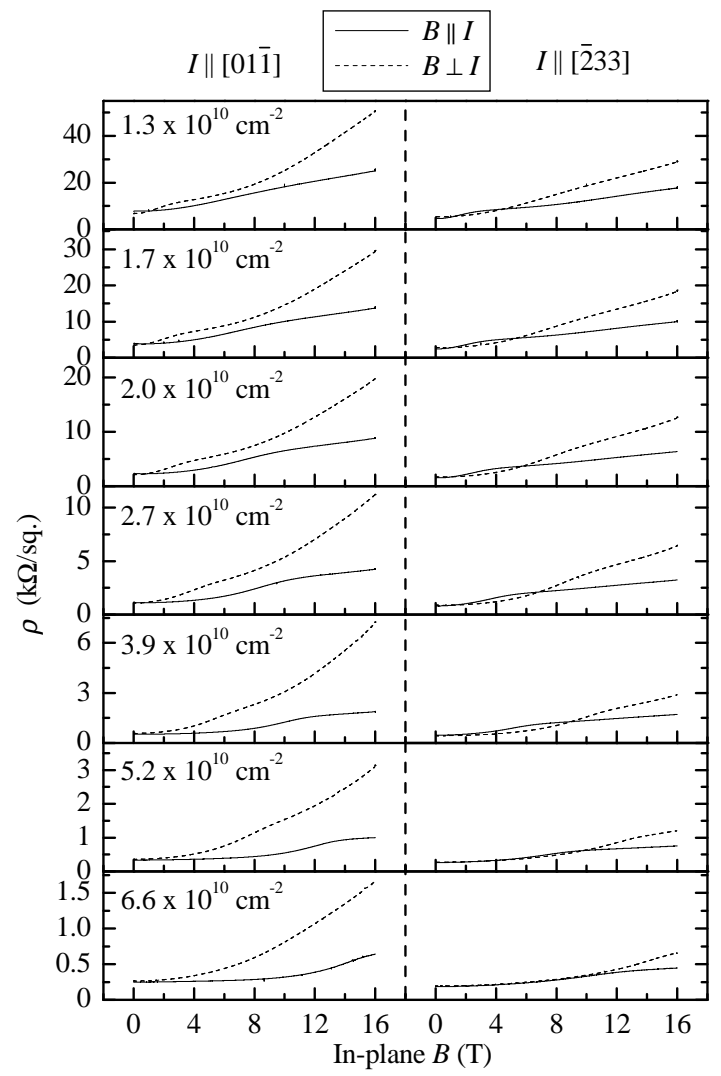

FIG. 2. Magnetoresistance at $T=0.3 \mathrm{~K}$ in an in-plane $B$, showing how resistivity changes as the orientation of $B$ is changed from parallel to perpendicular to the current $(I)$ direction. The density $p$ is listed in each panel.

slope, followed by another inflection point beyond which the traces curve upwards again. To highlight this behavior, the arrows in Fig. 3 are placed between the two inflection points, at a $B$ we will refer to as $B^{*}$. Surprisingly, for each $p, B^{*}$ for the $B \|[\overline{2} 33]$ traces is about $4 \mathrm{~T}$ smaller than for the $B \|[01 \overline{1}]$ traces, regardless of the $I$ direction. Also, $B^{*}$ becomes smaller as $p$ is reduced. Figure 3 reveals that the relative orientations of $B$ and the crystal axes play an important role in the MR features.

The existence of the MR features around $B^{*}$ is intriguing. Similar, though sharper, features have been observed in in-plane $B$ measurements in systems with multiple confinement subbands when a subband is depopulated [21]. We propose that the MR features observed in our data are related to the changes in the relative populations of the spin-subbands and the resulting changes in subband mobility and inter-subband scattering as the in-plane $B$ is increased. To test this hypothesis, we have done self-consistent subband calculations with no adjustable parameters 22] that give us spin-subband densities as a function of in-plane $B$ (Fig. 1). Figure 1 shows that the upper spin-subband de-populates more quickly for $B \|[\overline{2} 33]$ than for $B \|[01 \overline{1}]$, which can be traced back to the strong anisotropy of the 2DHS band structure in our system 23]. This is consistent 


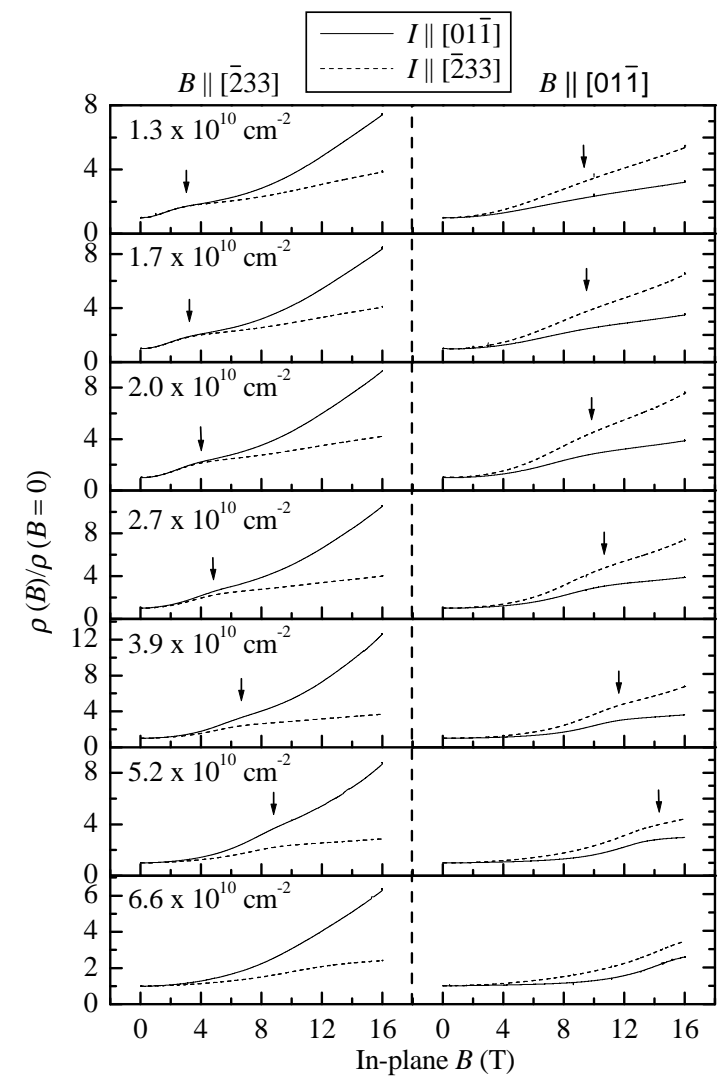

FIG. 3. Fractional change in resistivity due to an in-plane $B$, showing that the relative orientations of $B$ and the crystal axes play an important role in determining the position of the magnetoresistance features. The vertical arrows mark $B^{*}$ as defined in the text.

with the experimental observation that $B^{*}$ is smaller for $B \|[\overline{2} 33]$. Also, the $B$ at which the subband completely de-populates changes with $p$ in much the same way as $B^{*}$ does. However, $B^{*}$ is significantly smaller than the field at which the calculations show the upper spin-subband to reach zero density. We believe that the spin-subband de-population occurs at a lower $B$ than the band calculations predict because hole-hole interaction enhances the effective mass $m^{*}$ and effective $g$-factor $g^{*}$ in a dilute 2D system like ours. The average hole spacing in units of effective Bohr radius, $r_{s}$, for our experiment ranges from $r_{s}=6.9$ to 15.6 for $p=6.6 \times 10^{10} \mathrm{~cm}^{-2}$ to $1.3 \times 10^{10} \mathrm{~cm}^{-2}$ [24]. Okamoto et al. [16], conclude that for Si 2D electrons with $r_{s}$ in this range, $g^{*} m^{*}$ is enhanced by a factor of 2.7 to 5.5. Assuming similar enhancement in our samples, we would expect a reduction by the same factor of $B$ required to de-populate a subband. Using these numbers to adjust the de-population field given by the band calculations would put it near $B^{*}$, strongly suggesting that the observed MR features are due to spin-subband de-population.

Further evidence linking the MR features to spinsubband de-population is provided by our data at constant $p$ with changing $E_{\perp}$. The degree of asymmetry in

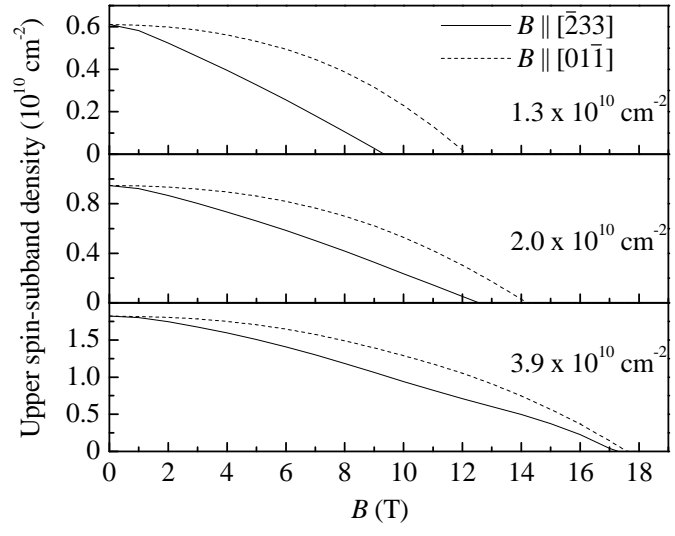

FIG. 4. Results of self-consistent calculations showing the upper spin-subband density with increasing in-plane $B$. Note the significant difference between curves for $B \|[\overline{2} 33]$ and for $B \|[01 \overline{1}]$.

the potential that confines the carriers to $2 \mathrm{D}$ controls the spin-splitting, and plays an important role in the magnitude of the $B=0$ temperature-dependence of the resistivity 10,11. For the data in Figs. 2 and $3, E_{\perp}$ is kept within $1 \mathrm{kV} / \mathrm{cm}$ of $5 \mathrm{kV} / \mathrm{cm}$. This $E_{\perp}$ is included in the calculations plotted in Fig. 4. Measurements at a constant $p=3.9 \times 10^{10} \mathrm{~cm}^{-2}$ as $E_{\perp}$ is increased from 4.5 $\mathrm{kV} / \mathrm{cm}$ to $12.5 \mathrm{kV} / \mathrm{cm}$ reveal that $B^{*}$ shifts to higher $B$ by about $2 \mathrm{~T}$. This observation is in agreement with the spin-subband de-population calculations done at fixed $p$ for varying $E_{\perp}$.

At higher in-plane $B$, beyond the MR features around $B^{*}$, the data in Fig. 3 are qualitatively similar. The traces for $B \perp I$ have greater slope than the corresponding traces with $B \| I$, regardless of crystal axes. In this regime the magnetic confinement can become comparable to the electric confinement, and the effects due to the finite-thickness of the 2DHS may be dominant. Indeed, Ref. [8] predicts that MR with in-plane $B$ should be significantly larger for $B \perp I$ than for $B \| I$, in agreement with our highest $B$ data. The data in which $E_{\perp}$ is changed at constant $p$ support this interpretation as well. As $E_{\perp}$ is increased, the confining potential becomes narrower, and the thickness of the 2DHS decreases. This should increase the $B$ required for finite-thickness effects to become important, and the data show that the MR anisotropy at $B=16 \mathrm{~T}$ is smaller for larger $E_{\perp}$.

We now turn to the $T$-dependence of MR to investigate the metallic phase in our $2 \mathrm{D}$ system. Figure a shows the $T$-dependence of MR at $p=3.9 \times 10^{10} \mathrm{~cm}^{-2}$, for the four measured relative orientations of $B, I$, and crystal axes. For each panel, the traces exhibit a nearly $T$-independent magnetic field $B_{T}$ which occurs near the trace's first inflection point. This is consistent with the data of Ref. [17]. For $B<B_{T}$, the data show metallic behavior, and for $B>B_{T}$, insulating behavior. $B_{T}$ is different in each 


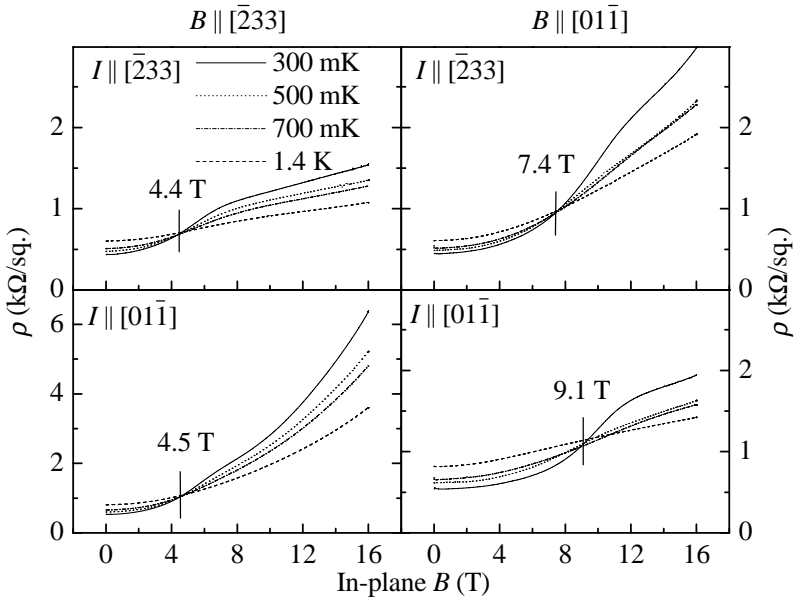

FIG. 5. Magnetoresistance data at various temperatures, for density $p=3.9 \times 10^{10} \mathrm{~cm}^{-2}$, for the four relative orientations of $B, I$, and crystal axes. The fields $B_{T}$ at which the resistivity is nearly $T$-independent are indicated by vertical marks.

panel and, similar to $B^{*}$, it changes much more for a rotation of the crystal axes relative to $B$ than it does for a rotation of $I$ relative to $B$. Our experiments indicate that $B^{*}$ and $B_{T}$ depend very similarly on the parameters of our systems $\left(p, E_{\perp}\right.$, direction of $\left.B\right)$. Our observation, which is in agreement with the in-plane MR data of Ref. [16], strongly suggests that the metallic behavior is linked to the presence of two populated spin-subbands [10,11,9.12.

In the data of Ref. [16], and likely in ours, the spinsubband de-population is linked to $B^{*}$ which is somewhat larger than $B_{T}$, so it appears that the metallic behavior changes to insulating before the upper spin-subband is fully de-populated. This may be because the low-density spin-subband stops playing a role in transport when its mobility $\mu$ becomes sufficiently low, before it is fully depopulated.

Finally, we note that Das Sarma and Hwang have recently reported calculations aiming to explain the $T$ dependence of the resistivity [7] and the in-plane MR \&] of $2 \mathrm{D}$ systems that exhibit metallic behavior at finite $T$. Their calculations, which include only charged impurity scattering and the orbital motion, qualitatively reproduce some of the experimental data. We wish to point out that our results reveal the importance of the spin degree of freedom, and suggest that for an understanding of the experimental data it is important to also consider a scattering mechanism involving the spin-subbands, perhaps intersubband scattering [9, 12]. Also important for (311)A GaAs 2D holes is the inclusion of interface roughness scattering: both the $T$-dependence of $\rho$ at $B=0$ (Fig. 1 $1 \mathrm{~b}$ ), as well as the in-plane MR data (Figs. 22 and 3), depend on the direction of the current in the crystal.

To summarize, our data reveal a surprising anisotropy of the in-plane magnetoresistance and its temperature dependence for GaAs (311)A 2D holes. The results show that the rate of the upper spin-subband's de-population with in-plane $B$ critically depends on the relative orientation of $B$ and the crystal axes. This points to the anisotropic nature of the $g$-factor and the spin-subband structure of GaAs (311)A 2D holes. Furthermore, we observe that the $B=0$ metallic behavior turns into insulating near $B$ at which the upper spin-subband depopulates. This observation, in agreement with the data for 2D electrons in Si [16], suggests that two spinsubbands are necessary for the expression of metallic behavior. These results also complement those of previous experiments [10, 11,9, 12 , which revealed that the presence of two spin-subbands with different populations appears to be linked to the metallic behavior.

This work was supported by the NSF and ARO. We thank M. Hofmann for stimulating discussions.

[1] E. Abrahams, P. W. Anderson, D. C. Licciardello, and T. V. Ramakrishnan, Phys. Rev. Lett. 42, 673 (1979).

[2] D. J. Bishop, D. C. Tsui, and R. C. Dynes, Phys. Rev. Lett. 44, 5737 (1980).

[3] S. V. Kravchenko, G. V. Kravchenko, and J. E. Furneaux, Phys. Rev. B 50, 8039 (1994); S. V. Kravchenko, D. Simonian, M. P. Sarachik, W. Mason, and J. E. Furneaux, Phys. Rev. Lett. 77, 4938 (1996).

[4] D. Popović, A. B. Fowler, and S. Washburn, Phys. Rev. Lett. 79, 1543 (1997); P. T. Coleridge, R. L. Williams, Y. Feng, and P. Zawadzki, Phys. Rev. B 56, R12764 (1997); J. Lam, M. D'Iorio, D. Brown, and H. Lafontaine, Phys. Rev. B 56, R12741 (1997); Y. Hanein et al., Phys. Rev. Lett. 80, 1288 (1998); M. Y. Simmons et al., Phys. Rev. Lett. 80, 1292 (1998); S. J. Papadakis and M. Shayegan, Phys. Rev. B 57, R15068 (1998).

[5] V. Dobrosavljević, E. Abrahams, E. Miranda, and S. Chakravarty, Phys. Rev. Lett. 79, 455 (1997); B. L. Altshuler and D. Maslov, Phys. Rev. Lett. 82, 145 (1999).

[6] V. M. Pudalov, Pis'ma Zh. Éksp. Teor. Fiz. 66, 168 (1997) [JETP Lett. 66, 175 (1997)].

[7] S. Das Sarma and E. H. Hwang, Phys. Rev. Lett. 83, 164 (1999).

[8] S. Das Sarma and E. H. Hwang, cond-mat/9909452.

[9] S. S. Murzin, S. I. Dorozhkin, G. Landwehr, and A. C. Gossard, JETP Lett. 67, 113 (1998).

[10] S. J. Papadakis et al., Science 283, 2056 (1999).

[11] S. J. Papadakis et al., Physica E 6, 284 (2000).

[12] Y. Yaish et al., cond-mat/9904324.

[13] V. M. Pudalov, G. Brunthaler, A. Prinz, and G. Bauer, , pis'ma Zh. Éksp. Teor. Fiz. 65, 168 (1997) [JETP Lett. 65, 932 (1997)].

[14] D. Simonian, S. V. Kravchenko, M. P. Sarachik, and V. M. Pudalov, Phys. Rev. Lett. 79, 2304 (1997).

[15] K. M. Mertes et al., Phys. Rev. B 60, R5093 (1999).

[16] T. Okamoto, K. Hosoya, S. Kawaji, and A. Yagi, Phys. Rev. Lett. 82, 3875 (1999). 
[17] J. Yoon et al., cond-mat/9907128.

[18] Another theoretical study predicts a qualitatively similar MR anisotropy due to spin-orbit coupling [G. H. Chen, M. E. Raikh, Y. S. Wu, cond-mat/9904451.

[19] J. J. Heremans, M. B. Santos, K. Hirakawa, and M. Shayegan, J. Appl. Phys. 76, 1980 (1994).

[20] M. Wassermeier et al., Phys. Rev. B 51, 14721 (1995).

[21] J. Jo et al., Phys. Rev. B 47, 4056 (1993).

[22] R. Winkler and U. Rössler, Phys. Rev. B 48, 8918 (1993); G. Goldoni and A. Fasolino, Phys. Rev. B 48, 4948 (1993).

[23] For $B=0$ the upper spin-subband density is smaller than half the $p$ because of the inversion-asymmetry-induced spin splitting of the subband states [9].

[24] To calculate $r_{s}$ we use an unenhanced $m^{*}=0.2 m_{e}$ [B. E. Cole et al., Phys. Rev. B 55, 2503 (1997)]. In our calculations we obtain a density-of-states $m^{*}$ at $B=0$ with approximately the same value. 